\title{
New Physics Search in Flavour Physics*
}

\author{
Tobias Hurth ${ }^{\mathrm{a}} \dagger$ \\ ${ }^{a}$ CERN, Dept. of Physics, Theory Unit, CH-1211 Geneva 23, Switzerland \\ SLAC, Stanford University, Stanford, CA 94309, USA
}

With the running $B$, kaon and neutrino physics experiments, flavour physics takes centre stage within today's particle physics. We discuss the opportunities offered by these experiments in our search for new physics beyond the SM and discuss their complementarity to collider physics.

We focus on rare $B$ and kaon decays, highlighting specific observables in an exemplary mode. We also comment on the so-called $B \rightarrow \pi \pi$ and $B \rightarrow K \pi$ puzzles. Moreover, we briefly discuss the restrictive role of long-distance strong interactions and some new tools such as QCD factorization and SCET to handle them.

\section{Introduction}

There are three main issues within the present $B$ and kaon physics programme: (i) the search for new degrees of freedom beyond the standard model (SM) in flavour- or CP-violating processes; (ii) the question of the precise mechanism of $\mathrm{CP}$ violation and (iii) the search for a quantitative understanding of the strong interactions within flavour observables.

Rare $B$ and kaon decays representing loopinduced processes are highly sensitive probes for new degrees of freedom beyond the SM. Through virtual (loop) contributions of new particles to such observables, one can investigate high-energy scales even before such energies are accessible at collider experiments.

Such flavour information is complementary to the collider data of the Large Hadron Collider (LHC); for example the present flavour data from the $B$ factories in the $b \rightarrow s$ sector is not very restrictive (yet). Within a supersymmetric new physics scenario, present bounds on squark mixing still allow for large contributions to flavourviolating squark decays at tree level, which can be measured at the LHC. In these cases, additional

${ }^{*}$ CERN-PH-TH/2005-168, SLAC-PUB-11530; based on invited talks given at Beauty 2005, the 10th International Conference on $B$-physics at Hadron Colliders, June 2024, Assisi (Perugia, Italy) and at QCD@Work, International Workshop on Quantum Chromodynamics, June 1620, Conversano (Bari, Italy)

${ }^{\dagger}$ Heisenberg Fellow information from future flavour experiments will be necessary to interpret those LHC data properly.

The CKM prescription of $\mathrm{CP}$ violation with one single phase is very predictive. Before the start of the $B$ factories, the neutral kaon system was the only environment where $\mathrm{CP}$ violation had been observed. It was difficult to decide if the CKM description of the SM really accounted quantitatively for the $\mathrm{CP}$ violation observed in the kaon system, because of the large theoretical uncertainties due to long-range strong interactions. The rich data sets from the present and planned $B$ experiments now allow for an independent and really quantitative test of the CKMinduced $\mathrm{CP}$-violating effects in several independent channels.

Quark-flavour physics is governed by the interplay of strong and weak interactions. One of the main difficulties in examining the observables in flavour physics is the influence of the long-distance strong interaction. The resulting hadronic uncertainties restrict the opportunities in flavour physics significantly. If new physics does not show up in $B$ physics through large deviations, as recent experimental data indicate, the focus on theoretically clean variables within the indirect search for new physics is mandatory.

Nevertheless there are new tools, such as QCD factorization and the soft-collinear effective theory (SCET), to tackle the strong interaction within $B$ decays. The large data sets from the 
$B$ experiments should be used to sharpen these new tools and improve our present understanding of the strong interaction.

\subsection{Experimental roadmap}

The present experimental roadmap of flavour physics offers great opportunities. Several $B$ physics experiments are successfully running at the moment and, in the upcoming years, new facilities will start to explore $B$ physics with increasing sensitivity and within various experimental settings. There are two $B$ factories, operating at the $\Upsilon(4 S)$ resonance in an asymmetric mode, successfully obtaining data, namely the BABAR experiment at SLAC (Stanford, USA) 1 and the BELLE experiment at KEK (Tsukuba, Japan) 2]. An upgrade of the BELLE machine is planned; and there are also plans for SuperBELLE 3. After the present hadronic B-physics programme at FERMILAB (Batavia, USA) 4, there are strong $B$-physics programmes planned at three LHC experiments at CERN in Geneva, especially at LHCb [5]. There is also a future option of a $B$-physics programme at a future linear collider via a Giga-Z factory (see 6] ).

The main motivation for a $B$ physics programme at hadron colliders is the huge $b$-quark production cross section with respect to the one at $e^{+} e^{-}$machines, and the opportunity to analyse also the $B_{s}$ system. Nevertheless, a future Super$B$ factory would be competitive but also complementary to the planned hadronic $B$ physics programme (see for details 78 ).

There are many further sectors of flavour physics that offer important experimental opportunities. $K$ decays such as $K \rightarrow \pi \nu \bar{\nu}$ and $K_{L} \rightarrow \pi^{0} \ell^{+} \ell^{-}$are extremely sensitive to possible new degrees of freedom and are largely unexplored. In fact, at present we have fewer constraints on short-distance-dominated $s \rightarrow d$ quark transitions than on $b \rightarrow s$ ones. In the presence of new physics, charm physics could provide important inputs by future $e^{+} e^{-}$and fixedtarget experiments. Searches for electric dipole moments of various particles are a very important source of information on the flavour and CP structure. Open questions in neutrino physics, regarding their masses, their mixing and their particle nature, are actively being attacked in the present and future experimental programme. The study of the correlation of neutrino properties with flavour phenomena in the chargedlepton and in the quark sector, e.g. chargedlepton flavour violation, is also an important target. Pushing the present limits on $\mu \leftrightarrow e$ and $\mu \leftrightarrow \tau$ transitions might lead to important insight.

\subsection{Hadronic uncertainties}

The crucial problem in the new physics search within flavour physics is the optimal separation of new physics effects and hadronic uncertainties. This can be successfully solved only for a selected number of golden observables in flavour physics, where hadronic physics can be disentangled to a large extent and clean tests of the SM are possible. In principle there are three strategies:

- One can focus on inclusive decays modes. These modes are dominated by the partonic contributions because bound-state effects of the final states are eliminated by averaging over a specific sum of hadronic states. Moreover, also longdistance effects of the initial state are accounted for, through the heavy mass expansion in which the inclusive decay rate of a heavy $B$ meson is calculated, using an expansion in inverse powers of the $b$-quark mass. In fact, one can use quark-hadron duality to derive a heavy mass expansion of the decay rates in powers of $\Lambda_{\mathrm{QCD}} / m_{b}$ (HME). For example, it turns out that the decay width of the $\bar{B} \rightarrow X_{s} \gamma$ is well approximated by the partonic decay rate, which can be calculated in renormalization-group-improved perturbation theory:

$\Gamma\left(\bar{B} \rightarrow X_{s} \gamma\right)=\Gamma\left(b \rightarrow X_{s}^{\text {parton }} \gamma\right)+\Delta^{\text {nonpert }}$

Non-perturbative corrections occur at the order $\Lambda_{Q C D}^{2} / m_{b}^{2}$ only. The absence of first-order power corrections is a consequence of the fact that there is no independent gauge-invariant operator of dimension 4 in the operator product expansion because of the equations of motion. The latter fact implies a rather small numerical impact of the non-perturbative corrections to the decay rate of inclusive modes. Nevertheless, there are additional nonperturbative corrections within 
inclusive modes due to necessary cuts in the experimental spectra like the photon energy spectrum in $\bar{B} \rightarrow X_{s} \gamma$ (see 9]).

- In exclusive processes, however, one cannot rely on quark-hadron duality and has to face the difficult task of estimating matrix elements between meson states. Therefore, exclusive modes are not well-suited to the new physics search in general. Nevertheless, one can focus on ratios of exclusive decay modes such as asymmetries, where large parts of the hadronic uncertainties partially cancel out. In particular, there are CP asymmetries that are governed by one weak phase only. In that specific case the hadronic matrix elements cancel out completely.

- There are also specific decays like $K \rightarrow \pi \nu \bar{\nu}$ modes where the hadronic uncertainties can be eliminated by experimental data. In these kaon decays the hadronic matrix element can be related to the well-known rare semileptonic $K_{l 3}$ decays.

Regarding the hadronic matrix elements of exclusive modes, the method of QCD-improved factorization has been systemized for non-leptonic decays in the heavy-quark limit. This method allows for a perturbative calculation of QCD corrections to naive factorization and is the basis for the up-to-date predictions for exclusive rare $B$ decays in general [10. However, within this approach, a general, quantitative method to estimate the important $1 / m_{b}$ corrections to the heavy-quark limit is missing.

A more general quantum field theoretical framework was proposed - known under the name of SCET - which allows for a deeper understanding of the QCD factorization approach 1112 . In contrast to the well-known heavy-quark effective theory (HQET), the recently proposed SCET does not correspond to a local operator expansion. While HQET is only applicable to $B$ decays, when the energy transfer to light hadrons is small, for example to $B \rightarrow D$ transitions at small recoil to the $D$ meson, it is not applicable, when some of the outgoing, light particles have momenta of order $m_{b}$; then one faces a multi scale problem:

a) $\Lambda=$ few $\times \Lambda_{\mathrm{QCD}}$, the soft scale set by the typical energies and momenta of the light degrees of freedom in the hadronic bound states; b) $m_{b}$ the hard scale set by the heavy-b-quark mass (we note, that in the $B$-meson rest frame, for $q^{2} \simeq 0$ also the energy of the final-state hadron is given by $E \simeq m_{b} / 2$ ); c) the hard-collinear scale $\mu_{\mathrm{hc}}=\sqrt{m_{b} \Lambda}$ appears through interactions between soft and energetic modes in the initial and final states. The dynamics of hard and hardcollinear modes can be described perturbatively in the heavy-quark limit $m_{b} \rightarrow \infty$.

The separation of the two perturbative scales from the non-perturbative hadronic dynamics is formalized, within the framework of SCET, with the small expansion parameter $\lambda=\sqrt{\Lambda / m_{b}}$. Thus, SCET describes $B$ decays to light hadrons with energies much larger than their masses, assuming that their constituents have momenta collinear to the hadron momentum. On a technical level, the implementation of power counting in $\lambda$, at the level of momenta, field and operators, corresponds directly to the well-known method of regions for Feynman diagrams [13].

The large varity of experimental data on those decay modes allows us to test these new tools and perhaps to reach sufficient accurary for the determination of the CKM parameters and even for the detection of new physics effects.

\section{Exploration of higher scales via rare de- cays}

Rare $B$ and kaon processes often represent flavour changing neutral currents (FCNCs) and occur in the SM only at the loop level. This fact leads to the high sensitivity to potential new degrees of freedom beyond the SM. Such potential new contributions are not suppressed with respect to the SM contributions (see Fig 1). This indirect

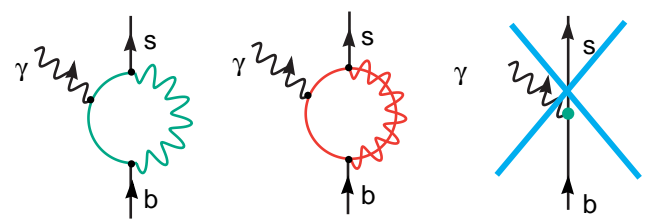

Figure 1. Standard and nonstandard contributions to $b \rightarrow s \gamma$ at the-loop level only . 
search for new physics signatures within flavour physics takes place today in complete darkness, given that we have no direct evidence of new particles beyond the SM. However, the day the existence of new degrees of freedom is established by the LHC, the searches for anomalous phenomena in the flavour sector will become mandatory. The problem then will no longer be to discover new physics, but to measure its (flavour) properties. In this context, the measurement of theoretically clean rare decays, even when found to be SM-like, will lead to important and valuable information of the structure of the new physics models and will lead to complementary information to the LHC collider data.

Because new physics effects beyond the SM seem to be rather small it is important to go beyond the pure study of branching ratios and also look at complex kinematic distributions, in particular at CP, forward-backward, isospin and polarization asymmetries. Only the measurements of a large overconstraining set of these observables allow us to detect specific pattern and to distinguish between various new-physics scenarios.

Finally, rare decays are also important tools to analyse the famous flavour problem, namely how FCNCs are suppressed beyond the SM. This problem has to be solved by any viable new physics model. One solution of the flavour problem is given by minimal flavour violation (MFV). In [27, a consistent definition of this scenario was presented, which essentially requires that all flavour and $\mathrm{CP}$-violating interactions be linked to the known structure of Yukawa couplings. The constraint within an effective field approach is introduced with the help of a symmetry concept and can be shown to be renormalization-groupinvariant 27.

Perhaps this MFV-based effective field theory approach is too pessimistic from the current point of view. One of the key predictions of the MFV is the direct link between the $b \rightarrow s, b \rightarrow d$, and $s \rightarrow d$ transitions. This prediction within the $\Delta F=1$ sector is definitely not well-tested at the moment and there is still room for new flavour structures to be discovered. Nevertheless, in contrast to the scale of the electroweak symmetry breaking, there is no similarly strong argument that new flavour structures have to appear at the electroweak scale.

\section{1. $b \rightarrow s / d \gamma$ and $b \rightarrow s \ell^{+} \ell^{-}$modes}

The inclusive $b \rightarrow s \gamma$ mode is still the most prominent rare decay, because it has already measured by several independent experiments [14 15 16 17 18 18 19] and the present experimental accuracy has reached the $10 \%$ level 20 :

$\mathrm{BR}\left[\bar{B} \rightarrow X_{s} \gamma\right]=(3.52 \pm 0.30) \times 10^{-4}$.

In the near future, more precise data on this mode are expected from the $B$ factories. Thus, it is mandatory to reduce the present theoretical uncertainty accordingly. A systematic improvement certainly consists in performing a complete NNLL calculation which will reduce the wellknown large uncertainty due to the definition of the charm mass 21] by a factor 2 as was recently shown [22. In a recent theoretical update of the NLL prediction of this branching ratio, the uncertainty related to the definition of $m_{c}$ was taken into account by varying $m_{c} / m_{b}$ in the conservative range $0.18 \leq m_{c} / m_{b} \leq 0.31$ which covers both, the pole mass (with its numerical error) value and the running mass $\bar{m}_{c}\left(\mu_{c}\right)$ value with $\mu_{c} \in\left[m_{c}, m_{b}\right]$ [24]: $\operatorname{BR}\left[\bar{B} \rightarrow X_{s} \gamma\right]=(3.70 \pm$ $\left.\left.0.35\right|_{m_{c} / m_{b}} \pm\left. 0.02\right|_{\mathrm{CKM}} \pm\left. 0.25\right|_{\text {param. }} \pm\left. 0.15\right|_{\text {scale }}\right) \times$ $10^{-4}$. The stringent bounds obtained from the $B \rightarrow X_{s} \gamma$ mode on various non-standard scenarios (see e.g. 2512627282930 are a clear example of the importance of clean FCNC observables in discriminating new-physics models.

Besides the $b \rightarrow s \gamma$ mode, also the $b \rightarrow s \ell^{+} \ell^{-}$ transitions are already accessible at the $B$ factories 313233 , inclusively and exclusively. Quite recently also the $b \rightarrow d \gamma$ transition was measured for the first time 34 (for a recent review see [35]). The inclusive decay $b \rightarrow s \ell^{+} \ell^{-}$is particularly attractive because of kinematic observables such as the invariant dilepton mass spectrum and the forward-backward (FB) asymmetry. This inclusive decay is also dominated by perturbative contributions if the $c \bar{c}$ resonances that show up as large peaks in the dilepton invariant mass spectrum are removed by appropriate kinematic cuts. In the 'perturbative windows', namely in the low- $s$ (dilepton mass) region 


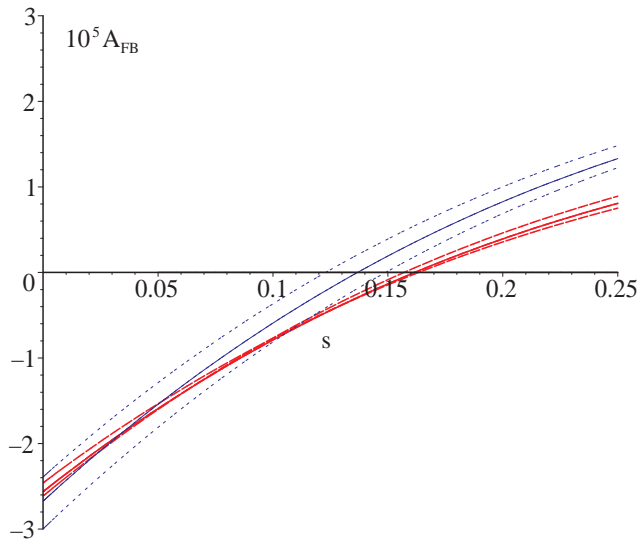

Figure 2. Comparison between NNLL and NLL results for $A_{F B}(s)$ in the low $s$ region. The three thick lines are the NNLL predictions for $\mu=5 \mathrm{GeV}$ (full), and $\mu=2.5$ and $10 \mathrm{GeV}$ (dashed); the dotted curves are the corresponding NLL results. All curves for $m_{c} / m_{b}=0.29$.

$\left(0.05<s=q^{2} / m_{b}^{2}<0.25\right.$ and also in the high- $s$ region with $0.65<s$, a theoretical precision comparable with the one reached in the decay $b \rightarrow s \gamma$ is in principle possible. The recently calculated NNLL contributions 363738394041 have significantly improved the sensitivity of the inclusive $\bar{B} \rightarrow X_{s} \ell^{+} \ell^{-}$decay in testing extensions of the SM in the sector of flavour dynamics, in particular the value of the dilepton invariant mass $\left(q_{0}^{2}\right)$, for which the differential forward-backward asymmetry vanishes, is one of the precise predictions in flavour physics (see Fig 2):

$q_{0, \mathrm{NNLL}}^{2}=(3.90 \pm 0.25) \mathrm{GeV}^{2}$.

Let us briefly comment on the impact of the exclusive $B \rightarrow K^{(*)} \ell^{+} \ell^{-}$modes. Hadronic uncertainties on these exclusive rates are dominated by the errors on form factors and are much larger than in the corresponding inclusive decays. In fact, following the analysis presented in Ref. [42, we see that inclusive modes already put much stronger constraints on the various Wilson coefficients. Concerning the measurement of a zero in the spectrum of the forward-backward asymmetry, things are different. According to Refs. 43] the value of the dilepton invariant mass $\left(q_{0}^{2}\right)$, for which the differential forward-backward asymmetry vanishes, can be predicted in quite a clean way. In the QCD factorization approach at leading order in $\Lambda_{\mathrm{QCD}} / m_{b}$, the value of $q_{0}^{2}$ is free from hadronic uncertainties at order $\alpha_{s}^{0}$ (a dependence on the soft form factor $\xi_{\perp}$ and the light cone wave functions of the $B$ and $K^{*}$ mesons appear at NLL). Within the SM, the authors of Ref. 43 . find: $q_{0}^{2}=(4.2 \pm 0.6) \mathrm{GeV}^{2}$. As in the inclusive case, such a measurement will have a huge phenomenological impact.

\subsection{There is also beauty in kaon physics}

Although the general focus within flavour physics is at present on $B$ systems, kaon physics offers interesting complementary opportunities in the new physics search, such as the exclusive rare decays $K^{+} \rightarrow \pi^{+} \nu \bar{\nu}$ and $K_{L} \rightarrow \pi^{0} \nu \bar{\nu}$. These decay modes are extremely sensitive to possible new degrees of freedom, but they also allow for an accurate determination of the unitarity triangle, which is completely independent from that of the $B$ system (for a recent review, see [44]).

These modes are basically unexplored yet. While there is only an upper limit on the neutral mode, three events were found in the charged mode by the AGS E787 and the E949 Collaborations at Brookhaven [4546, leading to

$\operatorname{BR}\left(K^{+} \rightarrow \pi^{+} \nu \bar{\nu}\right)=\left(1.47_{-0.9}^{+1.3}\right) \times 10^{-10}$.

Within the experimental and theoretical uncertainties this is fully consistent with the present theory prediction, which is based on a perturbative NNLL QCD analysis 47 and on a recent improvement of the long-distance contributions [4]:

$\operatorname{BR}\left(K^{+} \rightarrow \pi^{+} \nu \bar{\nu}\right)_{\mathrm{SM}}=(0.80 \pm 0.11) \times 10^{-10}$.

The error is dominated by parametrical errors on CKM matrix elements and on $m_{c}$ which can be significantly reduced in the future.

The rare decays $K^{+} \rightarrow \pi^{+} \nu \bar{\nu}$ and $K_{L} \rightarrow \pi^{0} \nu \bar{\nu}$ are both exceptionally clean modes, for two reasons essentially. First, the hard (quadratic) GIM mechanism, is active; thus, these decays are dominated by short-distance dynamics. In fact, at 
the quark level the two processes arise from the $s \rightarrow d \nu \bar{\nu}$ process, which originates from a combination of the $Z$ penguin and a double $W$ exchange (see Fig 31).

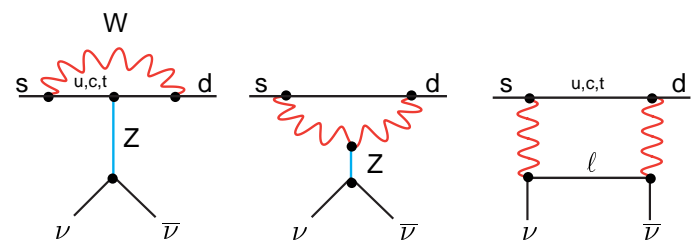

Figure 3. Graphs for $s \rightarrow d \nu \bar{\nu}$ in the SM

In these graphs the $u, c, t$ quarks appear as internal lines. The hard GIM mechanism implies on the amplitude level:

$A_{q} \sim m_{q}^{2} / m_{W}^{2} V_{q s}^{*} V_{q d}, q=u, c, t$.

Thus, the top-quark contribution dominates, with a smaller contribution, in the case of the $K^{+} \rightarrow$ $\pi^{+} \nu \bar{\nu}$ decay, from the charm contribution. The up-quark contribution is in both cases negligible, so that $s \rightarrow d \nu \bar{\nu}$ is essentially a short-distance process.

Moreover, the short-distance amplitude is governed by a single semileptonic operator whose hadronic matrix element can be determined experimentally by the semileptonic kaon decay $K^{+} \rightarrow \pi^{0} e^{+} \nu$ using isospin symmetry; so the main hadronic uncertainties can be eliminated by experimental data.

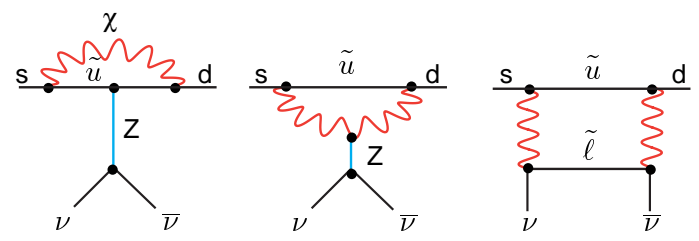

Figure 4. Graphs for $s \rightarrow d \nu \bar{\nu}$ in supersymmetry

Besides their rich CKM phenomenology, the decays $K_{L} \rightarrow \pi^{0} \nu \bar{\nu}$ and $K^{+} \rightarrow \pi^{+} \nu \bar{\nu}$, as loop-induced processes, are very sensitive to new physics beyond the SM and are crucial tools to discriminate between various new physics scenarios in the future. Thanks to the cleanliness of the theoretical predictions, the measurement of these decays leads to very accurate constraints on any new physics model and will help us to discriminate between various new physics scenarios in the future, when new physics is discovered within the direct search. Moreover, there are also very interesting and theoretically clean correlations between $B$ and $K$ physics allowing for crucial precision tests of the SM and also of so-called minimal flavour violation scenarios in which the flavour structure is essentially SM-like. These correlations are generally violated in models with new sources of flavor violation. There is also the possibility that these clean rare decay modes themselves lead to direct evidence for new physics, if the measured decay rates are not compatible with the SM. New effects in supersymmetric models, for example, can be induced through new boxand penguin-diagram contributions which involve new particles such as charged Higgs or charginos and stops (Fig. 4), that replace the $W$ boson and the up-type quark of the SM (Fig. 3).Explicit analyses of possible post-SM scenarios, with direct new-physics contributions in the $s \rightarrow d \bar{\nu} \nu$ amplitude or in $B \bar{B}$ mixing, can be found in 49 and 44 .

Besides the recently finalized Brookhaven experiments on the charged mode [456] and the running experiment on the neutral mode at KEK [51], there are two new proposals for the charged mode [52 53], one for the neutral [54] currently under discussion.

The novel feature of the proposed measurement of $K^{+} \rightarrow \pi^{+} \nu \bar{\nu}$ at CERN [53] is that, in contrast to previous experiments, one does not use stopped kaons but kaons in flight. One expects $80 \mathrm{~K}^{+} \rightarrow$ $\pi^{+} \nu \bar{\nu}$ events in about two years of data taking, starting in 2010, based on the SM predictions.

As can be seen in Fig. [5] the main background is the mode $K^{+} \rightarrow \pi^{+} \pi^{0}$. Thus, one of the main issues is the vetoing of the photons out of the $\pi^{0}$ decay, which is much easier than in previous experiments because of the high-energy kaon beam. 
New physics search in flavour physics

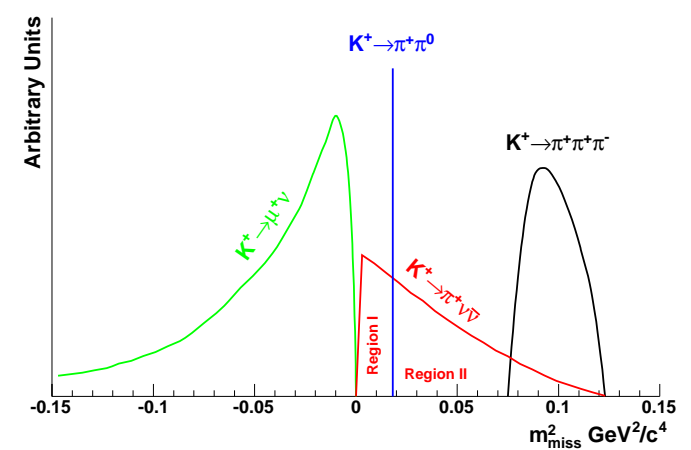

Figure 5. Distribution of the missing mass squared for the signal and the most frequent kaon decays. The signal region is divided into region I, which lies between the two prominent two-body decays of the charged kaon, while region II extends to the three-pion kinematical limit [50].

\subsection{Charmless rare $B$ decays: $B \rightarrow \pi \pi$}

Charmless rare $B$ decays are an ideal testing ground for the QCD factorization approach. The QCD factorization theorems on non-leptonic decay modes, first proposed in [10, identify shortdistance effects $\left(T_{\mathrm{I}}\right.$ and $\left.T_{I I}\right)$ that can be systematically calculated in perturbation theory: $\left\langle P P\left|H_{\mathrm{eff}}\right| B\right\rangle=F^{B \rightarrow P} \cdot T_{\mathrm{I}} \otimes \phi_{P}+T_{\mathrm{II}} \otimes \phi_{B} \otimes$ $\phi_{P} \otimes \phi_{P}+$ terms suppressed by $1 / m_{b}$, where the symbol $\otimes$ represents convolution with respect to the light-cone momentum fractions of light quarks inside the mesons. Non-perturbative effects are parametrized in terms of a few universal functions such as form factors $\left(F^{B \rightarrow P}\right)$ and light-cone distribution amplitudes $\left(\phi_{B}, \phi_{P}\right)$, on which our information is rather restricted. Moreover, phenomenological applications are limited by the insufficient information on (power-suppressed) nonfactorizable terms. Thus, the limiting factor of the QCD factorization theorems is twofold, namely the insufficient information on their nonperturbative input and on the power-suppressed (non-factorizable) terms. This problem should be tackled by the large data sets made available by current and future $B$ physics experiments and by the development of suitable new non-perturbative methods on the theory side.

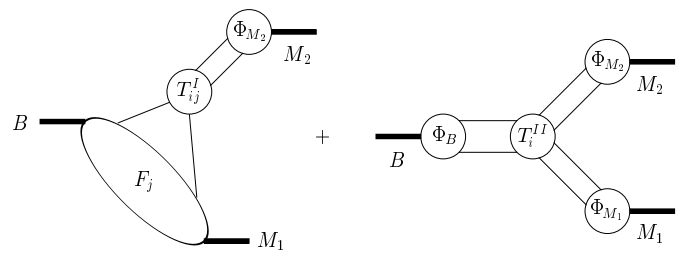

Figure 6. Illustration of QCD factorization theorems for non-leptonic $B$ decays

If one does not want to rely on particular assumptions about the hadronic dynamics, the traditional model-independent approach to charmless non-leptonic $B$-decays is to decompose the various decay amplitudes according to isospin 6869. The present experimental data on $B \rightarrow$ $\pi \pi$ decays is consistent with this isospin decomposition. In [55] it was proposed to use the modelindependent predictions from QCD factorization for the factorizable part of the decay amplitudes. The standard isospin analysis is then applied to the non-factorizable contributions only, which thus covers the model-dependent estimate of chirally enhanced power corrections, large deviations from the standard hadronic input parameters, etc. Comparing with the experimental data on branching ratios and $\mathrm{CP}$ asymmetries, this method allows to quantify the amount of nonfactorizable effects in particular isospin amplitudes without additional theoretical bias.

From the present data on $B \rightarrow \pi \pi$ decays, one finds that small non-factorizable contributions are disfavoured. Clearly, the dynamical origin of these non-factorizable corrections remains a theoretical challenge. At present, different phenomenological assumptions can accommodate the data. For instance, the latest update [57] of the BBNS approach [56 advocates scenarios where certain hadronic input parameters are put to the edges of the allowed regions and the estimate about the size of a particular class of $1 / m_{b}$ corrections is included in the theoretical uncertainties. Other authors assume the dynamical enhancement of certain flavour topologies to explain the experimental data, for instance the charming penguin approach of [58. Also a new analysis 
using SCET methods was recently presented [59, where SCET relations in leading order are combined with $S U(3)$ flavour relations. This leads to a further reduction of hadronic parameters due to the vanishing of some strong phases in the $m_{b} \rightarrow \infty$ limit.

Often only dominant $1 / m_{b}$ corrections are identified corresponding to simple $1 / m_{b}$ SCET operators. However, the $1 / m_{b}$ corrections also include more power-suppressed decay current operators and more insertions of subleading Lagrangian terms which lead to terms sensitive to higher Fock-state-contributions (see Fig[7) which are not necessarily suppressed.

Finally, it should be stressed that any dynamical assumption which further constrains the isospin analysis may lead to a strong bias when used in CKM fits. Therefore, one should rather use the experimental data itself to distinguish between different alternatives, and to measure the power-suppressed non-factorizable matrix elements in QCD factorization/SCET.
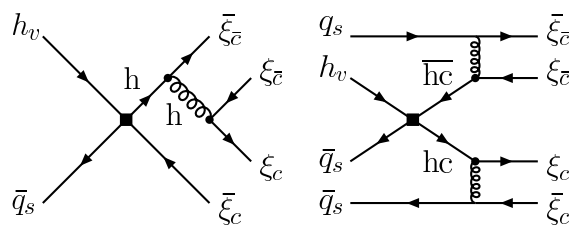

Figure 7. Annihilation contributions to $B \rightarrow P P$. (a) Example for one-gluon exchange included in the BBNS analysis. (b) Example for higher Fockstate-contribution

\subsection{Is there a $B \rightarrow K \pi$ puzzle?}

The $B \rightarrow K \pi$ modes are well-known for being sensitive to new electroweak penguins beyond the SM 6061. The present data on CP averaged $K \pi$ branching ratios can be expressed in terms of three ratios:

$$
\begin{array}{r}
R=\frac{\tau_{B^{+}}}{\tau_{B^{0}}} \frac{\mathrm{BR}\left[B_{d}^{0} \rightarrow \pi^{-} K^{+}\right]+\mathrm{BR}\left[\bar{B}_{d}^{0} \rightarrow \pi^{+} K^{-}\right]}{\operatorname{BR}\left[B_{d}^{+} \rightarrow \pi^{+} K^{0}\right]+\mathrm{BR}\left[B_{d}^{-} \rightarrow \pi^{-} \bar{K}^{0}\right]} \\
R_{n}=\frac{1}{2} \frac{\mathrm{BR}\left[B_{d}^{0} \rightarrow \pi^{-} K^{+}\right]+\mathrm{BR}\left[\bar{B}_{d}^{0} \rightarrow \pi^{+} K^{-}\right]}{\operatorname{BR}\left[B_{d}^{0} \rightarrow \pi^{0} K^{0}\right]+\operatorname{BR}\left[\bar{B}_{d}^{0} \rightarrow \pi^{0} \bar{K}^{0}\right]}
\end{array}
$$

$$
R_{c}=2 \frac{\mathrm{BR}\left[B_{d}^{+} \rightarrow \pi^{0} K^{+}\right]+\mathrm{BR}\left[B_{d}^{-} \rightarrow \pi^{0} K^{-}\right]}{\mathrm{BR}\left[B_{d}^{+} \rightarrow \pi^{+} K^{0}\right]+\mathrm{BR}\left[B_{d}^{-} \rightarrow \pi^{-} \bar{K}^{0}\right]}
$$

The present data 63 ,

$R=0.84_{-0.05}^{+0.06}, R_{n}=0.82_{-0.07}^{+0.08}, R_{c}=1.00_{-0.08}^{+0.09}$,

appears somewhat anomalous, when compared, for example, with the approximate sum rule proposed in 656466 which leads to the prediction $R_{c}=R_{n}$. The corresponding BBNS predictions 5767 are

$R=0.91_{-0.11}^{+0.13}, R_{n}=1.16_{-0.19}^{+0.22}, R_{c}=1.15_{-0.17}^{+0.19}$.

One can also use approximate flavour symmetries (isospin or $S U(3)_{F}$ ) to relate different decay amplitudes and reduce the number of unknown hadronic parameters 6869. This procedure is often combined with additional dynamical assumptions about the importance of certain flavour topologies that can be identified in the factorization approximation only. In particular, in a recent study [70] along these lines, the $B \rightarrow \pi \pi$ data was used to make theoretical predictions on the $B \rightarrow K \pi$ modes. This specific approach leads to

$R=0.94_{-0.03}^{+0.03}, R_{n}=1.14_{-0.07}^{+0.08}, R_{c}=1.11_{-0.07}^{+0.06}$.

At present, it is difficult to estimate the theoretical uncertainties due to the assumptions made in this approach, but in the future those assumptions including the $S U(3)_{F}$ symmetry can be tested experimentally.

Does this slightly anomalous behaviour guide us to new physics in the $K \pi$ modes? It is important to note that the deviation of $R_{n}$ and $R_{c}$ from unity is solely due to isospin-breaking effects 66 and the amount of short-distance isospin breaking in the $\mathrm{SM}$ is too small to explain the experimental number. Whereas the authors of [70 argue that this may point to an interesting avenue towards new physics in electroweak penguin operators, the collaboration in 62 considers this deviation as a statistical fluctuation, which is consistent with the SM - even when the $S U(3)_{F}$ constraints are enforced. Not surprisingly, the $B \rightarrow K \pi$ data has triggered several new-physics analyses.

Due to the large non-factorizable contributions identified in the $B \rightarrow \pi \pi$ channel, it is difficult to decide whether the data on $B \rightarrow \pi K$ 
decays point to new physics (for example to isospin breaking via new degrees of freedom as discussed in 6061), or whether they can be explained by non-factorizable $S U(3)_{F^{-}}$or isospinviolating QCD and QED effects within the SM. For the latter possibility, it was pointed out in [55] that the actual expansion parameter for nonperturbative isospin violating effects in $B \rightarrow \pi K$ can be as large as $5-10 \%$, for instance via non-factorizable corrections from CKM-enhanced long-distance QED penguins. This seems to be in the right ball park to at least partly explain the deviation of $R_{n}$ from unity.

Apart from the theoretical questions about the interpretation of the data, there are also some experimental issues:

- Radiative corrections to decays with charged particles in the final states had not been taken into account properly in previous analyses, an effect which was expected to lead to an increased branching ratio of these modes (see 62]) and which could bring $R_{n}$ closer to unity. In fact, quite recently such effects were calculated [71] and found to be not negligible. On the experimental side, BABAR has already taken into account these results on the final state interactions in order to estimate the efficiency by comparing with the used Monte Carlo program PHOTOS [72]. The new experimental branching ratios quoted by BABAR correspond now to a well-defined photon energy-cut. These corrections on the experimental side already led to a $+6 \%$ correction in the $K^{+} \pi^{-}$mode and $+8 \%$ in the $\pi^{+} \pi^{-}$mode 73 . However, the theoretical prediction still has to be corrected accordingly in order to be compared with the experimental data using a photon energy-cut. There is an additional effect of around $3-5 \%$ expected [7], but the precise value depends on the matching with the short-distance calculation.

- It has also been argued in 74 that the present pattern could perhaps result from underestimating the $\pi^{0}$ detection efficiency which implies an overestimate for any branching ratio involving a $\pi^{0}$. The authors of 74] propose therefore to consider the ratio $\left(R_{n} R_{c}\right)^{1 / 2}$ in which the $\pi^{0}$ detection efficiency cancels out. However, this idea has not found any confirmation on the experimental side.

One may also shed some light on the dynamical origin of non-factorizable effects, which may stimulate further studies with non-perturbative methods. In particular, a systematic classification of power-suppressed matrix elements from non-factorizable SCET operators should give an alternative scheme compared to the traditional classification in terms of flavour topologies.

\section{Search in CP-violating observables}

The CKM prescription of $\mathrm{CP}$ violation with a single phase is very predictive. It was proposed already in 1973 [75], before the experimental confirmation of the existence of the second family. Before the start of the $B$ factories, the neutral kaon system was the only environment where $\mathrm{CP}$ violation had been observed. It has been difficult to decide if the CKM description of the SM really accounted quantitatively for the $\mathrm{CP}$ violation observed in the kaon system, because of the large theoretical uncertainties due to long-range strong interactions. The rich data sets from the $B$ factories now allow for an independent and really quantitative test of the CKM-induced CP-violating effects in several independent channels. Within the golden $B$ mode, $B_{d} \rightarrow J / \psi K_{S}$, the CKM prescription of $\mathrm{CP}$ violation has already passed its first precision test; in fact, the measured $\mathrm{CP}$ violation is well in agreement with the CKM prediction 21. Nevertheless, there is still room for non-standard $\mathrm{CP}$ phases. An additional experimental test of the CKM mechanism is provided by the mode $B_{d} \rightarrow \Phi K_{S}$. This mode is induced at the loop level only and, therefore, it is much more sensitive to possible additional sources of $\mathrm{CP}$ violation than the tree-level-induced decay $B_{d} \rightarrow J / \psi K_{S}$. However, the poor statistics does not allow to draw final conclusions yet 21. We also note that the commonly used average over various $b \rightarrow s \bar{s} s$ modes are not a reasonable procedure because 


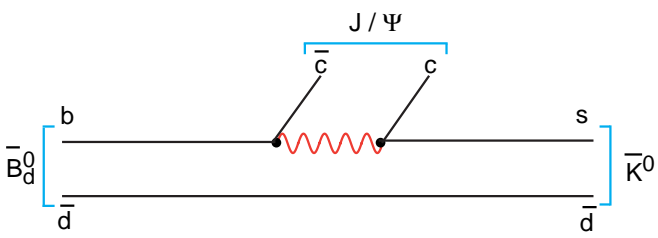

Figure 8. The tree-level process $B_{d} \rightarrow J / \psi K_{S}$

of the different hadronic uncertainties of those modes. There are also direct $\mathrm{CP}$ asymmetries

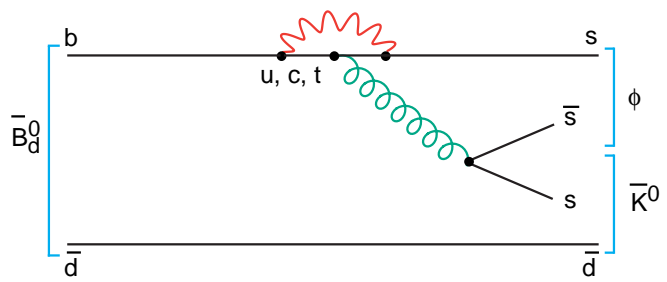

Figure 9. The loop-induced process $B_{d} \rightarrow \Phi K_{S}$

in loop-induced $\Delta F=1$ modes, which allow for additional precision tests of the mechanism of $\mathrm{CP}$ violation 767724 . Currently, these decays are less probed than $\Delta F=2$ transitions. However, very precise measurements of direct $\mathrm{CP}$ asymmetries in inclusive rare $B$ decays, such as $b \rightarrow s$ or $b \rightarrow d$ transitions, will be possible in the near future. A first measurement of the so-called untagged direct $\mathrm{CP}$ violation in the summed inclusive $b \rightarrow s+d \gamma$ was already presented by the BABAR collaboration [78.

\section{Interplay of flavour and collider physics}

Within the next decade an important interplay of flavour and collider physics will take place. As from the day the existence of new degrees of freedom is established by the LHC, the study of anomalous phenomena in the flavour sector will become an important tool for studying their phenomenology.

For example, within supersymmetric extensions of the SM, the measurement of the flavour structure is directly linked to the crucial question of the supersymmetry-breaking mechanism. Thus, the flavour sector is important in distinguishing between models of supersymmetry. This example nicely shows the obvious complementary nature of flavour physics and collider physics. At the LHC direct searches for supersymmetric particles are essential in establishing the existence of new physics. On the other hand, there are a variety of possibilities for the origin of flavour structures within supersymmetry. Flavour physics provides an important tool with which fundamental questions, such as how supersymmetry is broken, can be addressed.

Until now the focus within the direct search for supersymmetry has been mainly on flavour diagonal observables. But there are many obvious relations between non-diagonal collider and low-energy flavour observables. In [79, flavour violation in the squark decays of the second and third generations, were analysed, taking into account results from $B$ physics, in particular from the rare decay $b \rightarrow s \gamma$. It was shown that correlations between various squark decay modes can be used to obtain more precise information on various flavour-violating parameters.

Within the minimal supersymmetric standard model (MSSM), there are two new sources of FCNCs, namely new contributions, which are induced through the quark mixing as in the $\mathrm{SM}$ and generic supersymmetric contributions through the squark mixing. The structure of the MSSM does not explain the suppression of FCNC processes which is observed in experiments (supersymmetric flavour problem). The generic new sources of flavour violation that may be present in supersymmetric models, in addition to those enclosed in the CKM matrix, are a direct consequence of a possible disalignment of quarks and squarks. One has to consider the various contributions to the squark mass matrices

$$
\left(\begin{array}{cc}
M_{f, L L}^{2}+F / D_{f, L L} & M_{f, L R}^{2}+F_{f L R} \\
\left(M_{f, L R}^{2}\right)^{\dagger}+F_{f, R L} & M_{f, R R}^{2}+F / D_{f, R R}
\end{array}\right)
$$

where $f$ stands for up- or down-type squarks. The matrices $M_{u, L L}$ and $M_{d, L L}$ are related by $S U(2)_{L}$ gauge invariance. In the super-CKM 
basis, where the quark mass matrices are diagonal and the squarks are rotated in parallel to their superpartners, the relation reads as $K^{\dagger} M_{u, L L}^{2} K=M_{d, L L}^{2}=M_{Q}^{2}$. In this basis the $F$-terms $F_{f L L}, F_{f R L}, F_{f R R}$, as well as the $D$ terms $D_{f L L}$ and $D_{f R R}$, are diagonal. All the additional flavour structure of the squark sector is encoded in the soft SUSY-breaking terms $M_{Q}^{2}$, $M_{f, R R}^{2}$ and $M_{f, L R}^{2}$. These additional flavour structures induce flavour-violating couplings to the neutral gauginos and higgsinos in the mass eigenbasis, which give rise to additional contributions to observables in the $K$ - and $B$-meson sector.

At present, new physics contributions to $s \rightarrow d$ and $b \rightarrow d$ transitions are strongly constrained. In particular, the transitions between first- and second-generation quarks, namely FCNC processes in the $K$ system, are the most formidable tools to shape viable supersymmetric flavour models. Nevertheless, most of the phenomena involving $b \rightarrow s$ transitions are still largely unexplored and leave open the possibility of large new physics effects, in spite of the strong bound of the famous $\bar{B} \rightarrow X_{s} \gamma$ decay, which still gives the most stringent bounds in this sector. Nevertheless, additional experimental information from the $\bar{B} \rightarrow X_{s} \ell^{+} \ell^{-}$decay at the $B$ factories and new results on the $B_{s}-\bar{B}_{s}$ mixing at the Tevatron might change this situation in the near future.

Squarks can decay into quarks of all generations if the most general form of he squark mass matrix is considered. The most important decay modes for the example under study are:

$\tilde{u}_{i} \rightarrow u_{j} \tilde{\chi}_{k}^{0}, d_{j} \tilde{\chi}_{l}^{+}$
$\tilde{d}_{i} \rightarrow d_{j} \tilde{\chi}_{k}^{0}, u_{j} \tilde{\chi}_{l}^{-}$

with $i=1, . ., 6, j=1,2,3, k=1, . ., 4$ and $l=1,2$.

These decays are controlled by the same mixing matrices as the contributions to $b \rightarrow s \gamma$. As this decay mode restricts the size of some of the elements, the question arises regarding to which extent flavour-violating squark decays are also restricted. The following tables show that flavourviolating decay modes are hardly constrained by current data. We used the so-called Snowmass point SPS\#1a 80 as a specific example, which is characterized by $m_{0}=100 \mathrm{GeV}, m_{1 / 2}=$ $250 \mathrm{GeV}, A_{0}=-100 \mathrm{GeV}, \tan \beta=10$ and $\operatorname{sign}(\mu)=1$. At the electroweak scale one gets the following data: $M_{2}=192 \mathrm{GeV}, \mu=351 \mathrm{GeV}$, $m_{H^{+}}=396 \mathrm{GeV}, m_{\tilde{g}}=594 \mathrm{GeV}, m_{\tilde{t}_{1}}=$ $400 \mathrm{GeV}, m_{\tilde{t}_{2}}=590 \mathrm{GeV}, m_{\tilde{q}_{R}} \simeq 550 \mathrm{GeV}$, and $m_{\tilde{q}_{L}} \simeq 570 \mathrm{GeV}$. We concentrated on the mixing between the second and third generations and we used one point in the parameter space representing typical results fulfilling the $b \rightarrow s \gamma$ constraint based on a LL QCD analysis as given in 2829 . In Tables 10 and 2 we have collected the branching ratios of squarks that are larger than 1\%. Clearly, all considered particles have large flavour-changing decay modes.

The results demonstrate that large flavourchanging decays of squarks and gluinos are consistent with current data from the Tevatron and the $B$ factories. Such a situation makes life at the LHC potentially more interesting, but more difficult. It is clear that it would be complicated or even impossible to disentangle the various decay modes at the LHC; flavour tagging is not sufficient. In these cases, additional information from future flavour experiments will be necessary to interpret that LHC data properly.

\section{Outlook}

It is expected that the experiments at the LHC will lead to discoveries of new degrees of freedom at the $\mathrm{TeV}$ energy scale. The precise nature of this new physics is unknown, but it is strongly expected that it will answer some of the fundamental questions related to the origin of electroweak symmetry breaking. Independently of the nature of the new physics, a flavour physics program parallel to the LHC's will be crucial to disentangle the precise features of the newly uncovered phenomena and to discriminate between different new physics scenarios. In particular, the measurement of theoretically clean loop-induced rare $B$ - and $K$-meson decays as highly sensitive probes for new degrees of freedom beyond the SM will lead to important information complementary to collider data; there are important fundamental questions that will be addressed exclusively by future flavour experiments, for 
Table 1

Typical branching ratios (in \%) of $u$-type squarks

\begin{tabular}{|c|cc|cc|cc|cc|cc|cc|}
\hline & $\tilde{\chi}_{1}^{0} c$ & $\tilde{\chi}_{1}^{0} t$ & $\tilde{\chi}_{2}^{0} c$ & $\tilde{\chi}_{2}^{0} t$ & $\tilde{\chi}_{3}^{0} c$ & $\tilde{\chi}_{3}^{0} t$ & $\tilde{\chi}_{4}^{0} c$ & $\tilde{\chi}_{4}^{0} t$ & $\tilde{\chi}_{1}^{+} s$ & $\tilde{\chi}_{1}^{+} b$ & $\tilde{\chi}_{2}^{+} s$ & $\tilde{\chi}_{2}^{+} b$ \\
\hline$\tilde{u}_{1}$ & 4.7 & 18 & 5.2 & 9.6 & $6 \times 10^{-3}$ & 0 & 0.02 & 0 & 11.3 & 46.4 & $2 \times 10^{-3}$ & 4.7 \\
$\tilde{u}_{2}$ & 19.6 & 1.1 & 0.4 & 17.5 & $2 \times 10^{-2}$ & 0 & $6 \times 10^{-2}$ & 0 & 0.5 & 57.5 & $3 \times 10^{-3}$ & 2.9 \\
$\tilde{u}_{3}$ & 7.3 & 3.7 & 20 & 1.4 & $6 \times 10^{-2}$ & 0 & 0.6 & 0 & 40.3 & 3.1 & 1 & 18.5 \\
$\tilde{u}_{6}$ & 5.7 & 0.4 & 11.1 & 5.3 & $4 \times 10^{-2}$ & 5.7 & 0.6 & 13.2 & 22.9 & 13.1 & 0.6 & 8.0 \\
\hline
\end{tabular}

Table 2

Typical branching ratios (in \%) of $d$-type squarks

\begin{tabular}{|c|cc|cc|cc|cc|cc|cc|c|}
\hline & $\tilde{\chi}_{1}^{0} s$ & $\tilde{\chi}_{1}^{0} b$ & $\tilde{\chi}_{2}^{0} s$ & $\tilde{\chi}_{2}^{0} b$ & $\tilde{\chi}_{3}^{0} s$ & $\tilde{\chi}_{3}^{0} b$ & $\tilde{\chi}_{4}^{0} s$ & $\tilde{\chi}_{4}^{0} b$ & $\tilde{\chi}_{1}^{-} c$ & $\tilde{\chi}_{1}^{-} t$ & $\tilde{\chi}_{2}^{-} c$ & $\tilde{\chi}_{2}^{-} t$ & $\tilde{u}_{1} W^{-}$ \\
\hline$\tilde{d}_{1}$ & 1.2 & 5.7 & 8.4 & 30.6 & $2 \times 10^{-2}$ & 1.5 & 0.2 & 0.9 & 16.6 & 34.1 & 0.6 & 0 & 0 \\
$\tilde{d}_{2}$ & 17.4 & 5.8 & 5.1 & 15.7 & $7 \times 10^{-2}$ & 7.4 & 0.3 & 09.2 & 9.7 & 19.7 & 0.7 & 0 & 8.8 \\
$\tilde{d}_{4}$ & 14.7 & 21.7 & 11.3 & 2.2 & $5 \times 10^{-2}$ & 10.6 & 0.5 & 8.4 & 22.1 & 3.6 & 1.2 & 0 & 3.4 \\
$\tilde{d}_{6}$ & 1.7 & 0.5 & 20.5 & 6.9 & 0.1 & 0.9 & 1.2 & 1.3 & 40.3 & 10.2 & 3.4 & 11.1 & 1.8 \\
\hline
\end{tabular}

example how FCNCs are suppressed beyond the SM (flavour problem), if there exist new sources of flavour and $\mathrm{CP}$ violation beyond those in the $\mathrm{SM}$, if there is $\mathrm{CP}$ violation in the $\mathrm{QCD}$ gauge sector, how neutrino masses are generated, and what the relation between the flavour structure in the lepton and quark sectors is. All these questions include exciting options to learn something about physics at a scale much higher than our current experiments. Thus, a diversified and thorough experimental programme in flavour physics will continue to be an essential element for the understanding of nature.

\section{Acknowledgement}

I would like to thank Augusto Ceccucci, Markus Cristinziani, Carlo Dallapiccola, Jerome Charles, and Gino Isidori for useful discussions. I am also very grateful to Thorsten Feldmann for a careful and critical reading of the manuscript.

\section{REFERENCES}

1. http://www.slac.stanford.edu/BFROOT/

2. http://belle.kek.jp/

3. S. Hashimoto et al., KEK-REPORT-2004-4

4. K. Anikeev et al., arXiv:hep-ph/0201071

5. P. Ball et al., arXiv:hep-ph/0003238

6. A. Ali et al., arXiv:hep-ph/0012218

7. A. G. Akeroyd et al., arXiv:hep-ex/0406071

8. J. Hewett et al., arXiv:hep-ph/0503261
9. M. Neubert, Eur. Phys. J. C $40 \quad$ (2005) 165 arXiv:hep-ph/0408179.

10. M. Beneke et al., Phys. Rev. Lett. 83 (1999) 1914 arXiv:hep-ph/9905312.

11. C. W. Bauer et al., Phys. Rev. D 63 (2001) 114020 arXiv:hep-ph/0011336.

12. M. Beneke et al., Nucl. Phys. B 643 (2002) 431 arXiv:hep-ph/0206152.

13. M. Beneke and V. A. Smirnov, Nucl. Phys. B 522 (1998) 321 hep-ph/9711391.

14. R. Barate et al. [ALEPH Collaboration], Phys. Lett. B 429 (1998) 169.

15. K. Abe et al. [Belle Collaboration], Phys. Lett. B 511 (2001) 151, arXiv:hep-ex/0103042.

16. S. Chen et al. [CLEO Collaboration], Phys. Rev. Lett. 87 (2001) 251807, arXiv:hep-ex/0108032.

17. B. Aubert et al. [BaBar Collaboration], arXiv:hep-ex/0207074

18. B. Aubert et al. [BaBar Collaboration], arXiv:hep-ex/0207076

19. P. Koppenburg et al. [Belle Collaboration], Phys. Rev. Lett. 93 (2004) 061803 arXiv:hep-ex/0403004.

20. J. Alexander et al. [Heavy Flavor Averaging Group (HFAG)], arXiv:hep-ex/0412073

21. P. Gambino and M. Misiak, Nucl. Phys. B 611, 338 (2001) arXiv:hep-ph/0104034.

22. H. M. Asatrian, C. Greub, A. Hovhannisyan, T. Hurth and V. Poghosyan, Phys. Lett. B 619 (2005) 322 arXiv:hep-ph/0505068.

23. T. Hurth, Rev. Mod. Phys. 75 (2003) 1159 arXiv:hep-ph/0212304.

24. T. Hurth, E. Lunghi and W. Porod, Nucl. Phys. B 704 (2005) 56 arXiv:hep-ph/0312260.

25. G. Degrassi, P. Gambino and G. F. Giudice, JHEP 0012, 009 (2000) arXiv:hep-ph/0009337.

26. M. Carena, D. Garcia, U. Nierste and 
C. E. M. Wagner, Phys. Lett. B 499, 141 (2001) arXiv:hep-ph/0010003.

27. G. D'Ambrosio, G. F. Giudice, G. Isidori and A. Strumia, Nucl. Phys. B 645, 155 (2002) arXiv:hep-ph/0207036.

28. F. Borzumati, C. Greub, T. Hurth and D. Wyler, Phys. Rev. D 62, 075005 (2000) arXiv:hep-ph/9911245.

29. T. Besmer, C. Greub and T. Hurth, Nucl. Phys. B 609, 359 (2001) arXiv:hep-ph/0105292.

30. M. Ciuchini, E. Franco, A. Masiero and L. Silvestrini, Phys. Rev. D 67, 075016 (2003) [Erratum-ibid. D 68, 079901 (2003)] arXiv:hep-ph/0212397.

31. M. Iwasaki [Belle Collaboration], arXiv:hep-ex/0503044

32. B. Aubert et al. [BABAR Collaboration], Phys. Rev. Lett. 93 (2004) 081802 arXiv:hep-ex/0404006.

33. K. Abe et al. [The Belle Collaboration], arXiv:hep-ex/0508009

34. K. Abe et al., arXiv:hep-ex/0506079

35. T. Hurth and E. Lunghi, eConf C0304052 (2003) WG206 arXiv:hep-ph/0307142.

36. H. H. Asatryan, H. M. Asatrian, C. Greub and M. Walker, Phys. Rev. D 65 (2002) 074004 arXiv:hep-ph/0109140.

37. A. Ghinculov, T. Hurth, G. Isidori and Y. P. Yao, Nucl. Phys. B 648 (2003) 254 arXiv:hep-ph/0208088.

38. A. Ghinculov, T. Hurth, G. Isidori and Y. P. Yao, Nucl. Phys. B 685 (2004) 351 arXiv:hep-ph/0312128.

39. H. M. Asatrian, K. Bieri, C. Greub and A. Hovhannisyan, Phys. Rev. D $66 \quad$ (2002) 094013 arXiv:hep-ph/0209006.

40. C. Bobeth, M. Misiak and J. Urban, Nucl. Phys. B 574 (2000) 291 arXiv:hep-ph/9910220.

41. C. Bobeth, P. Gambino, M. Gorbahn and U. Haisch, JHEP 0404, 071 (2004) arXiv:hep-ph/0312090.

42. A. Ali, E. Lunghi, C. Greub and G. Hiller, Phys. Rev. D 66 (2002) 034002. arXiv:hep-ph/0112300.

43. M. Beneke, T. Feldmann and D. Seidel, Nucl. Phys. B 612 (2001) 25 arXiv:hep-ph/0106067.

44. A. J. Buras, F. Schwab and S. Uhlig, arXiv:hep-ph/0405132

45. S. Adler et al. [E787 Collaboration], Phys. Rev. D 70 (2004) 037102 arXiv:hep-ex/0403034.

46. V. V. Anisimovsky et al. [E949 Collaboration], Phys. Rev. Lett. 93 (2004) 031801 arXiv:hep-ex/0403036.

47. A. J. Buras et al., arXiv:hep-ph/0508165

48. G. Isidori, F. Mescia and C. Smith, Nucl. Phys. B 718 (2005) 319 arXiv:hep-ph/0503107.

49. G. D'Ambrosio and G. Isidori, Phys. Lett. B 530 (2002) 108 arXiv:hep-ph/0112135.

50. Courtesy of Giuseppe Ruggiero

51. http://psux1.kek.jp/ e391/

52. http://www-ps.kek.jp/jhf-np/LOIlist/pdf/L04.pdf

53. http://na48.web.cern.ch/NA48/NA48-3/

54. http://www-ps.kek.jp/jhf-np/LOIlist/pdf/L05.pdf

55. T. Feldmann and T. Hurth, JHEP 0411 (2004) 037 arXiv:hep-ph/0408188.
56. M. Beneke et al., Nucl. Phys. B 606 (2001) 245 arXiv:hep-ph/0104110.

57. M. Beneke and M. Neubert, Nucl. Phys. B 675 (2003) 333 arXiv:hep-ph/0308039.

58. M. Ciuchini et al., Phys. Lett. B 515 (2001) 33 arXiv:hep-ph/0104126.

59. C. W. Bauer, I. Z. Rothstein and I. W. Stewart, arXiv:hep-ph/0510241

60. R. Fleischer and T. Mannel, arXiv:hep-ph/9706261

61. Y. Grossman, M. Neubert and A. L. Kagan, JHEP 9910, 029 (1999) arXiv:hep-ph/9909297.

62. J. Charles et al., Eur. Phys. J. C 41, 1 (2005) arXiv:hep-ph/0406184.

63. http://www.slac.stanford.edu/xorg/hfag/

64. M. Gronau and J. L. Rosner, Phys. Rev. D 59 (1999) 113002 arXiv:hep-ph/9809384.

65. H. J. Lipkin, Phys. Lett. B 445 (1999) 403 arXiv:hep-ph/9810351.

66. J. Matias, Phys. Lett. B 520 (2001) 131 arXiv:hep-ph/0105103.

67. M. Beneke private communication.

68. M. Gronau and D. London, Phys. Rev. Lett. 65 (1990) 3381.

69. Y. Nir and H. R. Quinn, Phys. Rev. Lett. 67 (1991) 541.

70. A. J. Buras et al., Nucl. Phys. B 697 (2004) 133 arXiv:hep-ph/0402112.

71. E. Baracchini and G. Isidori, arXiv:hep-ph/0508071

72. E. Barberio and Z. Was, Comput. Phys. Commun. 79 (1994) 291.

73. B. Aubert et al. [BABAR Collaboration], arXiv:hep-ex/0508046

74. M. Gronau and J. L. Rosner, Phys. Lett. B 572 (2003) 43 arXiv:hep-ph/0307095.

75. M. Kobayashi and T. Maskawa, Prog. Theor. Phys. 49 (1973) 652.

76. T. Hurth and T. Mannel, Phys. Lett. B 511 (2001) 196 arXiv:hep-ph/0103331.

77. T. Hurth and T. Mannel, AIP Conf. Proc. 602 (2001) 212 arXiv:hep-ph/0109041.

78. B. Aubert et al. [BaBar Collaboration], arXiv:hep-ex/0507001

79. T. Hurth and W. Porod, Eur. Phys. J. C 33 (2004) S764 arXiv:hep-ph/0311075.

80. B. C. Allanach et al., Eur. Phys. J. C 25 (2002) 113 [eConf C010630 (2001) $\mathrm{P} 125]$ arXiv:hep-ph/0202233. 